\title{
¿Se comporta el alfabetismo financiero como un bien económico?
}

\author{
Rubén Castro y Andrés Fortunato
}

RESUMEN

El alfabetismo financiero (AF) se entiende generalmente como un bien económico cuyo consumo el individuo decide sobre la base de su aporte esperado en la toma de decisiones financieras. Sin embargo, este marco conceptual no ha sido puesto a prueba empíricamente. En este trabajo se analiza la variación en el AF de individuos que experimentan eventos del ciclo de vida, observables en los datos y de presumible repercusión en las finanzas personales. El análisis de un panel de aproximadamente 12.000 individuos muestra que de 17 eventos seleccionados, 13 evidencian una correlación con las decisiones financieras, pero solo uno de ellos, la capacitación laboral, resulta asociado a un cambio en el AF. Esta evidencia pone en tela de juicio la conceptualización del AF como bien económico, y se suma a un grupo de trabajos que, por una u otra razón, han cuestionado la solidez conceptual del AF. 


\section{I}

\section{Introducción}

Los mercados financieros son cada vez más complejos y un número cada vez mayor de personas tienen acceso a ellos. En ese contexto, se presume que la capacidad de los individuos para optimizar sus finanzas tiene un papel sustantivo en su bienestar (véase, por ejemplo, Hilgert, Hogarth y Beverly, 2003; Campbell y otros, 2011, entre otros). De aquí surge el concepto de "alfabetismo financiero" (AF), como una característica de los individuos que es determinante de su capacidad de optimización financiera.

Aunque existen críticas sobre la coherencia de las distintas aproximaciones empíricas empleadas para medir el AF de los individuos, puede decirse que los niveles de AF en la población son sustancialmente más bajos de lo que parece recomendable (Hogarth y Hilgerth, 2002; Miles, 2004; Christelis, Jappelli y Padula, 2005; Lusardi y Mitchell, 2007a y 2007b; Lusardi, Mitchell y Curto, 2010; Landerretche y Martínez, 2011; Van Rooij, Lusardi y Alessie, 2011; Stone y Neumann, 2012, entre otros). Esto se observa consistentemente en todos los países de los que se tienen datos, especialmente entre los segmentos de población con menos recursos y entre las mujeres. Se ha propuesto que esta carencia de AF tiene no solo un impacto negativo individual, sino también en los mercados y en las recientes crisis financieras globales (Gerardi, Goette y Meier, 2010). De ahí que en muchos países se hayan implementado programas destinados a aumentar el nivel de AF en su población, iniciativa en la que subyace naturalmente la idea de que los beneficios sociales de estas intervenciones compensan holgadamente sus costos.

Sin embargo, la evaluación de impacto de estas intervenciones en el comportamiento financiero no arroja resultados claros (Lyons y otros, 2006; Hathaway y Khatiwada, 2008; Servon y Kaestnert, 2008; Willis, 2009, Mandell y Klein, 2009, entre otros). Varios autores responsabilizan de ello a la falta de un marco conceptual en la literatura sobre AF (Mason y Wilson, 2000; Willis, 2008; Remund, 2010, y Huston, 2010).

Para contar con mejores políticas y evaluaciones de impacto es necesario entender mejor el proceso de

$\square$ Marcela Urbina, Olivia Mitchell, Ximena Quintanilla y Eduardo Fajnzylber prestaron una gran ayuda a este proyecto. acumulación y desacumulación de AF (ADAF). Hasta ahora, salvo en un par de ejemplos (Delavande, Rohwedder y Willis, 2008, y Agarwal y otros, 2009), no se ha estudiado en profundidad la evolución del AF a lo largo del ciclo de vida de los individuos, o a través del tiempo, o frente a cambios en el entorno.

En la literatura no existe un consenso respecto de la conceptualización del AF (Huston, 2010). Mason y Wilson (2000) se preguntan por el significado del alfabetismo financiero, en tanto que Remund (2010), refiriéndose al concepto, señala que sus defensores han empleado la frase para describir conocimiento, habilidades, confianza y motivación necesarios para el manejo efectivo de dinero. Por cierto, existen diversas definiciones de AF (incluidos las habilidades numéricas, los conocimientos y los comportamientos financieros) y poca claridad sobre el proceso mismo de toma de decisiones financieras en el que se enmarca ${ }^{1}$.

En la aproximación más común en la literatura, el AF se entiende como un bien económico cuya acumulación el individuo optimiza en virtud del aporte esperado al proceso de toma de decisiones. En ese sentido, se adopta implícitamente un modelo de AF como "bien de información" (Bates, 1990), aunque también hay autores que adoptan un modelo de capital humano (véase, por ejemplo, Delavande, Rohwedder y Willis, 2008). En ambas aproximaciones subyace la idea del AF como bien económico cuyo consumo el individuo decide sobre la base de un proceso de optimización. La dinámica de acumulación y desacumulación de AF (ADAF) estaría condicionada entonces por el beneficio y costo esperado de adquirir AF. Si el beneficio esperado sube, o el costo baja, el individuo debiera adquirir más AF. De acá se deriva, por ejemplo, la idea de difundir la importancia del AF y de bajar los costos de acceso y el

\footnotetext{
${ }^{1}$ Van Rooij, Lusardi y Alessie (2011), por ejemplo, emplean preguntas que permiten evaluar la habilidad aritmética y los conocimientos básicos sobre el funcionamiento de las tasas de inflación y de interés, así como otras orientadas a medir conocimientos más avanzados relacionados con instrumentos del mercado financiero (acciones, bonos y fondos mutuos). Lusardi y Mitchel (2006), y Stone y Neumann (2012) utilizan una medida de preparación para el retiro. Lusardi (2008) usa el conocimiento sobre conceptos financieros básicos. Fajnzylber, Plaza y Reyes (2009) y Hastings y Tejeda-Ashton (2008) se enfocan en variaciones en la cantidad o formato de la información financiera entregada a los individuos.
} 
esfuerzo necesario para adquirir AF. El presente estudio se refiere a esta visión como "modelo económico del AF".

Pero - pese a la amplia utilización de este enfoqueno se cuenta con una evaluación empírica del ajuste entre el modelo económico del AF y la dinámica de ADAF.

El objetivo central de este trabajo es realizar dicha evaluación. En la perspectiva del modelo económico del AF, la ocurrencia de un evento que tiene implicancias para las finanzas de largo plazo de un individuo sube el valor esperado del AF, puesto que la incorporación de información nueva (la ocurrencia del evento) puede requerir tomar decisiones financieras. Si la dicha ocurrencia es exógena con respecto al AF, debiera observarse que este último aumenta en respuesta al evento en cuestión. Para ello se estima el impacto de la ocurrencia de eventos financieramente importantes en el AF de los individuos, sobre la base de una muestra representativa de la población chilena observada desde el año 2004 hasta el año 2009. La muestra corresponde a la Encuesta de Protección Social (EPS), un panel de cuatro rondas $(2002,2004,2006$ y 2009) más una quinta ronda, no disponible aún, efectuada en el año 2012. En estos datos longitudinales se incluye un módulo sobre conocimiento y habilidades financieras.

Los resultados de este estudio indican que no hay una variación significativa y consistente en el AF cuando ocurre un evento con sustanciales implicancias financieras. Se concluye entonces que el AF no se comporta como un bien económico.

En la siguiente sección se presentan los datos, la selección de eventos, los indicadores de AF y los análisis estadísticos utilizados en este trabajo. Luego, en las secciones III y IV se entregan los resultados y su discusión respectivamente.

\section{II}

\section{Metodología y datos}

En el modelo económico del AF, el beneficio de este se entiende como el impacto esperado que este tiene en la toma de decisiones financieras. Si los individuos experimentan un cambio en su trayectoria esperada de ingresos y gastos, y por lo tanto tienen un estímulo importante para reevaluar su situación financiera, también experimentan un alza en el beneficio esperado de adquirir AF. Ahora bien, si al mismo tiempo el costo de adquirir AF se mantiene constante, debiera observarse que en este proceso los individuos adquieren más AF. Una medición del acervo de AF antes y después del cambio en la trayectoria esperada de ingresos y gastos debiera, en estas circunstancias, reflejar un efecto positivo.

En este estudio se seleccionaron una serie de eventos observables, utilizando datos de encuestas en los que es razonable suponer que los individuos enfrentan cambios sustantivos en su flujo esperado de ingresos y egresos. Estos eventos conllevan profundos efectos multidimensionales en la vida de los individuos, como son los cambios en el estado civil, la salud, la capacitación laboral y la composición del hogar. Es poco probable que sean precisamente los cambios en el AF los que generen estas transiciones, por lo que es razonable asumir que estas son exógenas con respecto al AF. También es razonable asumir que, dado el intervalo de tiempo entre una encuesta y otra (dos años), los individuos hayan podido, en promedio, solucionar restricciones de tiempo, evitando alzas en el costo de adquirir AF. Bajo estos supuestos, se debiera observar alguna correlación positiva entre los eventos y el comportamiento financiero.

La metodología se basa en realizar la regresión de un indicador de AF con la ocurrencia de estos eventos, controlando por efectos fijos a nivel de individuos y por variables que cambian en el tiempo. Se utilizan datos de panel para una muestra de aproximadamente 14.000 individuos durante siete años. Los eventos se preseleccionan y luego se mantienen aquellos que muestran una correlación con los cambios en el "portfolio" financiero de los individuos. Además de efectos fijos a nivel de individuos, en el modelo econométrico se incorpora la variación en los ingresos de las personas como variable de control y se realiza un análisis independiente de cada subgrupo de edad, sexo o educación.

Existe una razón adicional para estudiar los eventos seleccionados en este trabajo. Son eventos que normalmente ocurren en coordinación con los organismos del Estado, lo que facilita la aplicación de políticas públicas en materia de finanzas personales. Por ello es especialmente relevante conocer la dinámica del AF en torno de estos eventos. Además, ellos pueden crear momentos receptivos (teachable moments), como se ha denominado a ciertas oportunidades de políticas de uso 
frecuente en salud y educación (Hansen, 1998; Syvertzen, Stout y Flanagan, 2009; Demark-Wahnefried y otros, 2005; McBride, Emmons y Lipkus, 2003; McBride y Ostroff, 2003, entre otros), y propuesto también para el campo del AF (Willis, 2008; GAO, 2004; Mandell y Klein, 2007 y 2009). La idea de estos momentos receptivos es que los individuos pasan por momentos de extrema receptividad y búsqueda activa de información.

\section{Datos}

Los datos utilizados en este estudio provienen de una encuesta longitudinal llamada Encuesta de Protección Social (EPS), que es llevada a cabo aproximadamente cada 2 años con el objetivo de obtener información sobre la operación y evolución del sistema de protección social en Chile (Bravo y otros, 2004). En la presente investigación se utilizan datos provenientes de las tres últimas rondas de EPS disponibles, efectuadas los años 2004, 2006 y 2009. En la ronda previa, realizada en 2002, se utilizó un cuestionario sustancialmente diferente a los de las rondas siguientes y, por lo tanto, no permite construir medidas comparables para variables como ingreso y gasto. Una breve descripción cuantitativa de la base se expone en el cuadro 1.

La primera ronda de la EPS, realizada entre junio de 2002 y enero de 2003, es representativa de los afiliados al sistema de pensiones a nivel nacional. La muestra consta de 17.246 individuos. En la segunda encuesta, que tuvo lugar entre noviembre de 2004 y mayo de 2005, se incluyó una muestra de aproximadamente 3.000 individuos no afiliados al sistema de pensiones. En la tercera y cuarta ronda, 2006 y 2009 respectivamente, solo se entrevistó a personas encuestadas en alguna de las rondas anteriores. En 2006 se incluyó un nuevo módulo de conocimiento financiero y habilidades no cognitivas.

Balanceando el panel para las tres últimas encuestas, se tiene una muestra con un total de 12.223 observaciones por ronda, de las cuales $5.905(48,3 \%)$ son hombres y 6.318 son mujeres $(51,7 \%)$. La distribución de la muestra por tramo etario y nivel educacional se aprecia en el cuadro 1.

Número de observaciones por ronda de EPS, 2004-2009

\begin{tabular}{|c|c|c|c|c|c|c|c|c|c|c|c|c|}
\hline & \multicolumn{4}{|c|}{2004} & \multicolumn{4}{|c|}{2006} & \multicolumn{4}{|c|}{2009} \\
\hline & \multicolumn{2}{|c|}{ Total } & \multicolumn{2}{|c|}{$\begin{array}{l}\text { Actualmente } \\
\text { cotizando }\end{array}$} & \multicolumn{2}{|c|}{ Total } & \multicolumn{2}{|c|}{$\begin{array}{l}\text { Actualmente } \\
\text { cotizando }\end{array}$} & \multicolumn{2}{|c|}{ Total } & \multicolumn{2}{|c|}{$\begin{array}{l}\text { Actualmente } \\
\text { cotizando }\end{array}$} \\
\hline & Cantidad & Porcentaje & Cantidad & Porcentaje & Cantidad & Porcentaje & Cantidad & Porcentaje & Cantidad & Porcentaje & Cantidad & Porcentaje \\
\hline Hombres & 5905 & 48,3 & 4346 & 54,6 & 5905 & 48,3 & 4200 & 55,1 & 5905 & 48,3 & 4442 & 54,5 \\
\hline Mujeres & 6318 & 51,7 & 3611 & 45,4 & 6318 & 51,7 & 3423 & 44,9 & 6318 & 51,7 & 3699 & 45,4 \\
\hline Edad $<35$ & 1663 & 13,6 & 1092 & 13,7 & 1358 & 11,1 & 973 & 12,8 & 976 & 8 & 790 & 9,7 \\
\hline $34<$ edad $<55$ & 5040 & 41,2 & 3737 & 47 & 4786 & 39,2 & 3453 & 45,3 & 4321 & 35,4 & 3351 & 41,2 \\
\hline $54<$ edad & 5522 & 45,2 & 3130 & 39,3 & 6081 & 49,8 & 3198 & 41,9 & 6928 & 56,7 & 4002 & 49,1 \\
\hline Educ $<=12$ & 9990 & 81,7 & 6122 & 76,9 & 9935 & 81,3 & 5765 & 75,6 & 9951 & 81,4 & 6177 & 75,9 \\
\hline $12<$ educ & 2235 & 18,3 & 1837 & 23,1 & 2290 & 18,7 & 1859 & 24,4 & 2274 & 18,6 & 1966 & 24,1 \\
\hline Total & 12223 & 100 & 7959 & 100 & 12223 & 100 & 7624 & 100 & 12223 & 100 & 8143 & 100 \\
\hline
\end{tabular}

Fuente: elaboración propia sobre la base de la Encuesta de Protección Social (EPS). 


\section{Selección de eventos}

Los eventos seleccionados son aquellos que: i) su sola materialización está presumiblemente ligada a una reevaluación de las finanzas de largo plazo; ii) son visibles en los datos, y iii) muestran una correlación significativa con los cambios en el consumo de bienes financieros.

Se preseleccionaron una serie de eventos. En cada par de rondas consecutivas (2004-2006 y 2006-2009) se codificó la ocurrencia de los eventos como uno o cero, dependiendo de si el evento correspondiente sucedió o no. Esta preselección inicial consta de 17 eventos:

1. Tener un hijo

2. Que alguien (que no sea el entrevistado) se jubile en el hogar

3. Casarse

4. Divorciarse

5. Enviudar

6. Obtener un título profesional

7. Obtener un diplomado

8. Realizar un curso de capacitación o perfeccionamiento laboral

9. Aprender un oficio

10. Obtener el primer trabajo permanente

11. Quedar cesante

12. Salir de un período de cesantía

13. Jubilar

14. Pasar a ser discapacitado

15. Dejar de ser discapacitado

16. Empeorar el estado de salud

17. Mejorar el estado de salud

Las correspondientes frecuencias de ocurrencia de cada uno de estos eventos, para cada par de rondas consecutivas y por categoría, pueden encontrarse en el cuadro 2. Los 17 eventos pueden agruparse en seis categorías: cambios en la estructura del hogar, cambios en el estado civil, cambio en el estatus de educación, capacitación, cambios en el estado laboral y cambios en salud.

El siguiente paso consiste en confirmar que los eventos considerados impliquen efectivamente algún cambio en el comportamiento financiero. Para ello se midió la correlación entre la ocurrencia de los eventos y los cambios en cuatro variables que implican alguna interacción del individuo con el sistema financiero. Estas variables son: i) cambios en la tasa de ahorro; ii) cambios en la deuda total sobre el ingreso; iii) cambios en los seguros de salud, y iv) cambios en la cantidad de seguros.
El modelo econométrico utilizado para buscar correlaciones es un modelo lineal de efecto fijo. De esta forma, cualquier variable estática omitida que no interactúe con las variables dinámicas no influye en los resultados. La incidencia de fenómenos homogéneos, causados por un efecto de la ronda o del tiempo, es parcialmente capturada por la constante:

$$
\begin{gathered}
\Delta Y_{i t}=\sum_{j=1}^{17} \beta_{j} \Delta X_{i j t}+\Delta \text { ingreso }_{i t}+\Delta \text { ingreso_hogar }_{i t} \\
+d_{\text {regiónit }}+d_{34}+\delta+\Delta \varepsilon_{i t}
\end{gathered}
$$

donde $Y$ corresponde a la variable de interacción con el sistema financiero, $X$ al vector de 17 eventos, $\delta$ a la constante, $i=1 \ldots N$ indica al individuo, $d_{34}$ es una dummy que indica si la diferencia es en las rondas 2006-2009 en lugar de las 2004-2006, $d_{\text {región }}$ es una dummy que captura heterogeneidad temporal por región, $\Delta i n g r e s o$ es la variación en el logaritmo del ingreso del entrevistado entre rondas, $\Delta i n g r e s o \_h o g a r$ es la variación del logaritmo del ingreso del resto de los habitantes del hogar, y $t=1,2$ corresponde a los períodos 2004-2006 y 2006-2009, respectivamente. Se asume que para todo el resto de observables y no observables las variables se mantienen lo suficientemente fijas como para ser eliminadas del modelo, o cambian con el tiempo de manera similar para todos los individuos, siendo incorporadas en la constante. También se asumen los restantes supuestos en Liker, Augustyniak y Duncan (1985) para la obtención de estimadores consistentes e insesgados.

Los resultados de estas regresiones se exponen en el cuadro 3. Cada una de las cuatro variables que recogen la interacción con el sistema financiero se analiza separadamente.

Para seleccionar el grupo de eventos finales, el criterio utilizado es la existencia de una correlación significativa de al menos un $10 \%$ entre el evento y alguno de los indicadores de interacción con el mercado financiero. En este ejercicio quedan descartados inmediatamente cuatro eventos: alguien se jubila en el hogar, divorciarse y los dos eventos de cambio en el estado laboral.

Ante la sospecha de multicolinealidad se inspeccionaron las correlaciones entre eventos descartando el problema. Todas resultaron ser menores de 0,1 , salvo en un par de casos en el segundo período donde apenas superaron esa magnitud. 


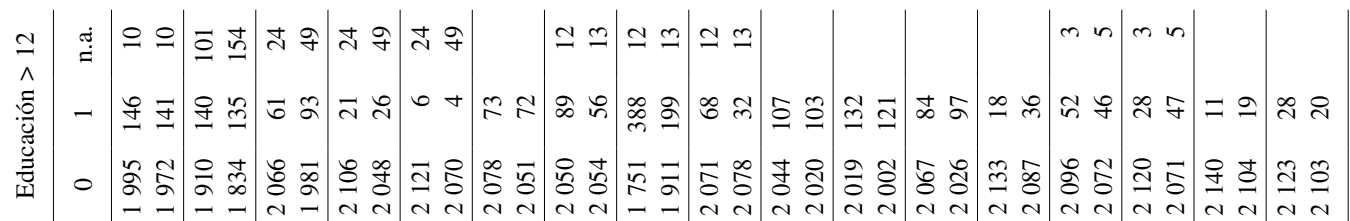

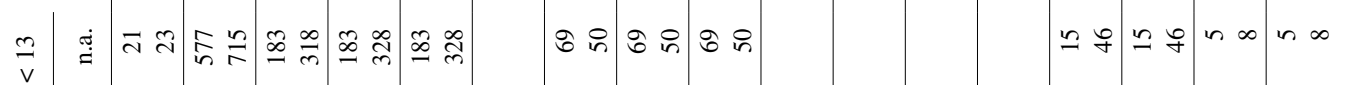

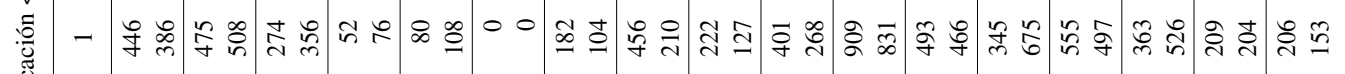
㽦 象 若 蛋

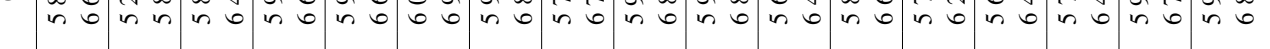

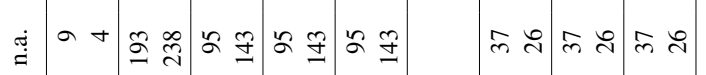

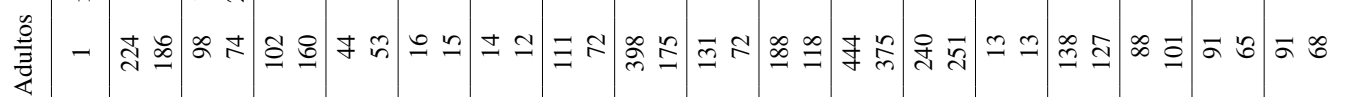

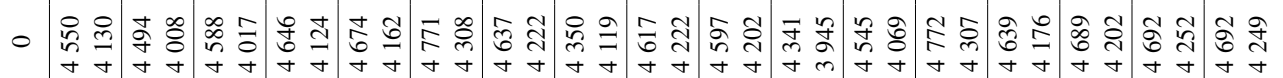

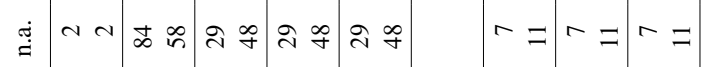

产

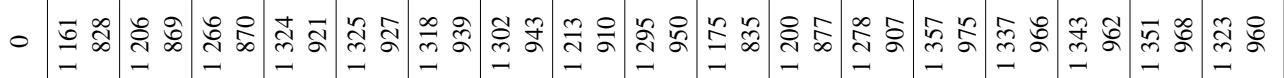

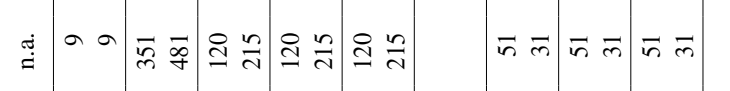

豙

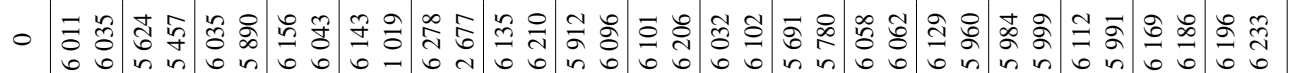

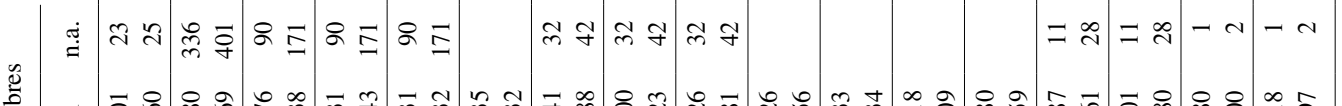

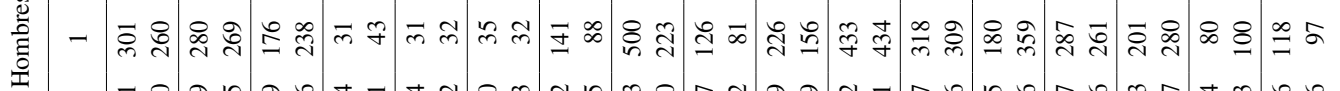

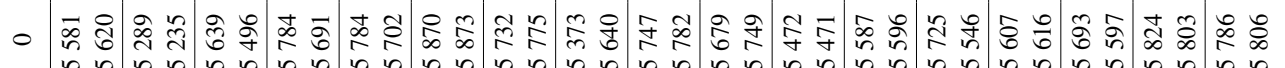

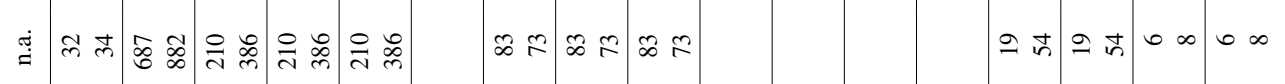



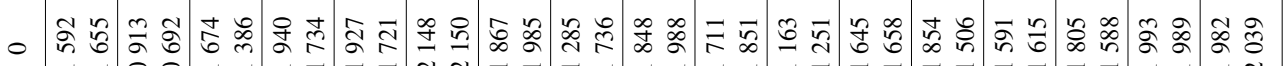

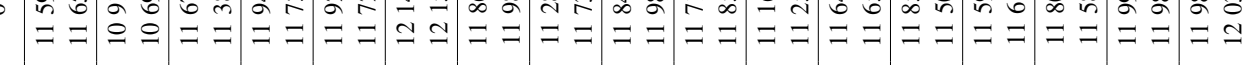

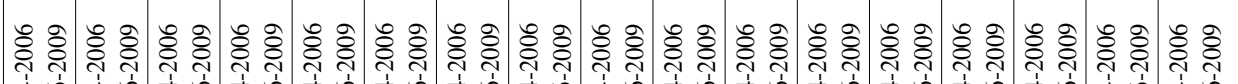
至

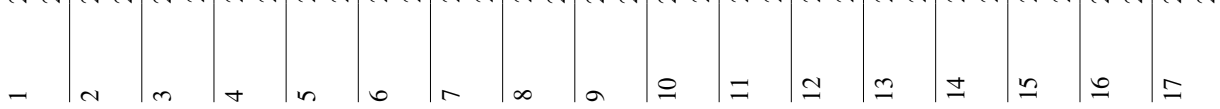
要 
Regresiones en primeras diferencias, indicadores de interacción con mercados financieros en comparación con selección preliminar de eventos, 2006-2009

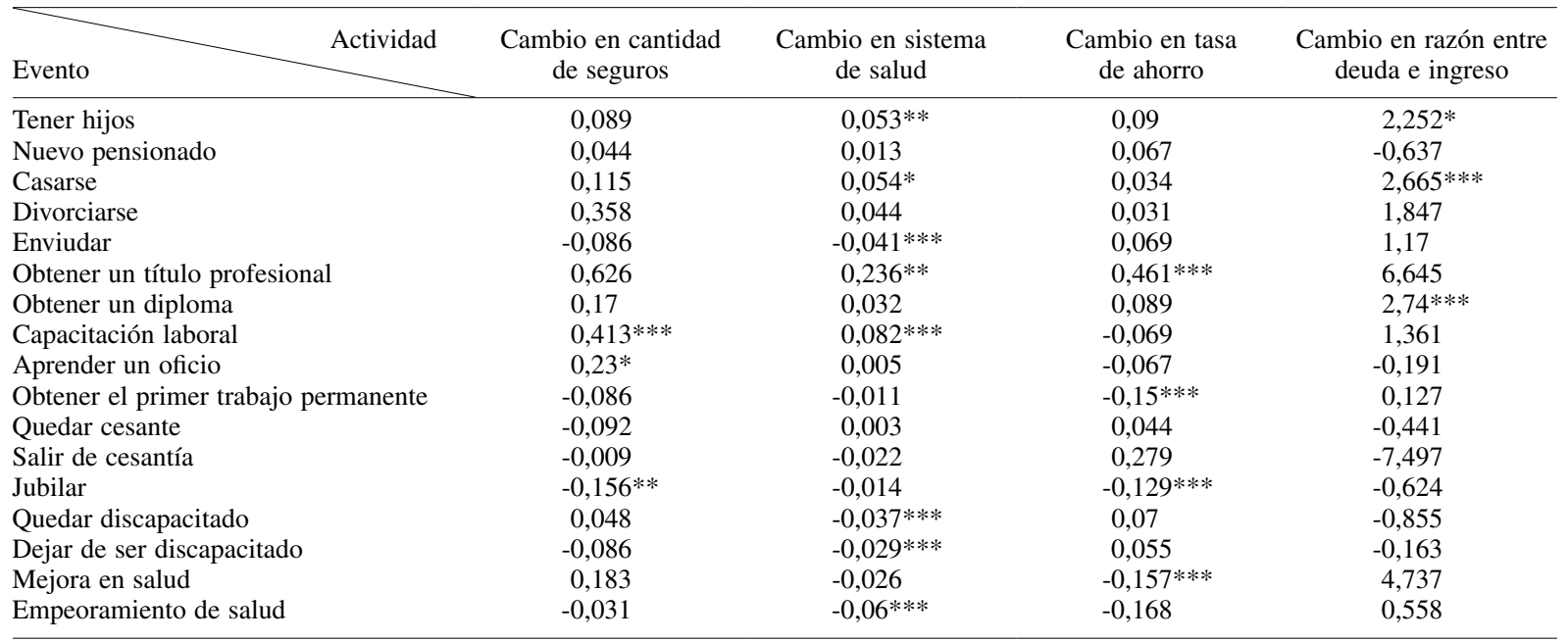

Fuente: elaboración propia sobre la base de la Encuesta de Protección Social (EPS).

$*$ significativo al $10 \%$; $* *$ significativo al $5 \%$; *** significativo al $1 \%$.

\section{Indicadores de AF}

Como medidas del acervo de AF de los individuos se utilizan dos indicadores. Uno de habilidades financieras básicas (HFB), construido con información disponible en las últimas dos rondas de la encuesta, y otro relacionado con el conocimiento sobre el sistema de pensiones (CSP) disponible en las tres últimas rondas. Con este último indicador se espera capturar una dimensión diferente de AF y además replicar el experimento realizado con HFB y extenderlo para la ronda 2004.

a) Medición de habilidades financieras básicas (HFB) El indicador utilizado para medir HFB se calcula para las rondas 2006 y 2009, empleando una serie de seis preguntas incluidas en un submódulo cuyo objetivo es medir la habilidad para entender o resolver cálculos matemáticos y financieros básicos. Las preguntas son las siguientes:

1. Si la posibilidad de contraer una enfermedad es de un $10 \%$, ¿cuántas personas de 1.000 contraerían la enfermedad?

2. Si cinco personas tienen los números premiados de la lotería y el premio es de dos millones de pesos, ¿cuánto recibiría cada una?

3. Suponga que Ud. tiene $\$ 100$ en una cuenta de ahorro, y la tasa de interés que gana por estos ahorros es de un $2 \%$ por año. Si mantiene el dinero por cinco años en la cuenta, ¿cuánto tendría al término de estos 5 años? (4 alternativas de rango).
4. Digamos que Ud. tiene $\$ 200$ en una cuenta de ahorro. La cuenta acumula $10 \%$ de intereses por año, ¿cuánto tendrá al cabo de dos años?

5. Suponga que Ud. posee $\$ 100$ en una cuenta de ahorro que entrega un interés de $1 \%$ anual. La tasa de inflación es de $2 \%$ anual. Si retira el dinero al cabo de un año usted podrá comprar: i) más de $\$ 100$; ii) exactamente $\$ 100$; iii) menos de $\$ 100$; iv) no sabe o no responde.

6. La siguiente oración ¿es verdadera o falsa?: "Comprar una acción de una empresa es menos riesgoso que comprar con el mismo dinero varias acciones en distintas empresas".

Cada respuesta es comparada con la respuesta correcta para obtener variables binarias (sabe/no sabe). Una descripción cuantitativa de las respuestas a cada pregunta, para la totalidad de la muestra, puede encontrarse en la parte superior del cuadro 4. Los hombres presentan una mayor cantidad de respuestas correctas en todas las preguntas para ambas rondas. En general, los jóvenes denotan también tener mejores resultados en todas las preguntas, salvo en la pregunta sobre inflación en 2009, donde el punto más alto se presenta en los adultos. Las personas con mayor educación también registran mejores resultados que sus contrapartes, las diferencias más contundentes se encuentran en las primeras tres preguntas donde la diferencia en el porcentaje de respuestas correctas entre ambos grupos supera incluso el $30 \%$. Con respecto a diferencias entre las rondas, las preguntas 2, 4, 5 y 6 ofrecen mejores resultados en general el año 2006, 
CUADRO 4

Habilidades financieras básicas: porcentaje de respuestas correctas por ronda y cohorte

(En porcentajes)

\begin{tabular}{|c|c|c|c|c|c|c|c|c|c|}
\hline Pregunta & Ronda & Total & Hombre & Mujer & Edad $<35$ & $34<$ edad $<55$ & $54<$ edad & Educ $<=12$ & $12<$ educ \\
\hline & & \multicolumn{8}{|c|}{ Total de la muestra } \\
\hline \multirow[t]{2}{*}{1} & 2006 & 44,3 & 49,8 & 39,4 & 60,0 & 46,7 & 39,0 & 37,9 & 73,0 \\
\hline & 2009 & 44,4 & 50,0 & 39,0 & 65,4 & 48,0 & 39,2 & 37,7 & 76,0 \\
\hline \multirow[t]{2}{*}{2} & 2006 & 40,4 & 45,0 & 36,0 & 48,6 & 42,1 & 37,3 & 35,7 & 62,0 \\
\hline & 2009 & 38,4 & 43,1 & 34,0 & 51,9 & 41,8 & 34,5 & 33,2 & 63,6 \\
\hline \multirow[t]{2}{*}{3} & 2006 & 45,7 & 49,5 & 42,2 & 57,9 & 47,7 & 41,6 & 40,6 & 69,0 \\
\hline & 2009 & 47,1 & 51,1 & 43,2 & 63,5 & 50,8 & 42,5 & 41,5 & 72,9 \\
\hline \multirow[t]{2}{*}{4} & 2006 & 1,7 & 2,3 & 1,1 & 2,4 & 1,8 & 1,5 & 0,7 & 6,1 \\
\hline & 2009 & 1,3 & 2,0 & 0,6 & 2,2 & 1,4 & 1,0 & 0,5 & 4,9 \\
\hline \multirow[t]{2}{*}{5} & 2006 & 25,2 & 27,5 & 23,0 & 27,1 & 25,3 & 24,7 & 22,2 & 38,4 \\
\hline & 2009 & 17,8 & 20,0 & 15,8 & 17,8 & 19,5 & 16,8 & 15,3 & 30,3 \\
\hline \multirow[t]{3}{*}{6} & 2006 & 43,6 & 46,0 & 41,3 & 49,7 & 45,5 & 40,7 & 40,2 & 59,5 \\
\hline & 2009 & 40,4 & 43,2 & 37,7 & 48,3 & 45,1 & 36,4 & 37,1 & 55,9 \\
\hline & & \multicolumn{8}{|c|}{ Solo personas que actualmente cotizan } \\
\hline \multirow[t]{2}{*}{1} & 2006 & 51,9 & 54,6 & 48,6 & 62,0 & 51,9 & 48,9 & 45,0 & 73,3 \\
\hline & 2009 & 52,3 & 55,9 & 48,0 & 67,7 & 51,8 & 49,9 & 45,2 & 76,7 \\
\hline \multirow[t]{2}{*}{2} & 2006 & 45,9 & 48,6 & 42,6 & 49,6 & 45,9 & 44,9 & 40,7 & 62,5 \\
\hline & 2009 & 44,5 & 47,0 & 41,5 & 53,4 & 45,2 & 42,3 & 38,9 & 63,8 \\
\hline \multirow[t]{2}{*}{3} & 2006 & 51,6 & 53,9 & 48,9 & 59,0 & 51,5 & 49,6 & 45,9 & 69,4 \\
\hline & 2009 & 54,9 & 56,8 & 52,6 & 66,5 & 55,1 & 52,6 & 49,0 & 74,3 \\
\hline \multirow[t]{2}{*}{4} & 2006 & 2,1 & 2,6 & 1,6 & 1,9 & 2,1 & 2,2 & 0,9 & 5,9 \\
\hline & 2009 & 1,6 & 2,3 & 0,8 & 2,2 & 1,7 & 1,5 & 0,6 & 4,9 \\
\hline \multirow[t]{2}{*}{5} & 2006 & 27,5 & 29,1 & 25,5 & 27,3 & 26,7 & 28,3 & 23,8 & 38,7 \\
\hline & 2009 & 20,2 & 21,8 & 18,3 & 18,4 & 20,9 & 19,9 & 17,2 & 30,2 \\
\hline \multirow[t]{2}{*}{6} & 2006 & 47,3 & 48,6 & 45,6 & 50,7 & 47,2 & 46,3 & 43,3 & 60,0 \\
\hline & 2009 & 44,9 & 46,3 & 43,2 & 48,1 & 47,4 & 42,2 & 41,5 & 55,9 \\
\hline
\end{tabular}

Fuente: elaboración propia sobre la base de la Encuesta de Protección Social (EPS).

mientras que las preguntas 1 y 3 aparecen con un mejor rendimiento el año 2009; sin embargo, las diferencias son en general menores de un 5\%, salvo en la pregunta 5 (sobre inflación) donde alcanzan un $7 \%$.

La misma información es presentada en la parte inferior del cuadro 4, pero utilizando solo respuestas de personas que se encuentran cotizando en el momento de la entrevista. En general, las diferencias entre rondas y categorías se mantienen tal como en el caso anterior, pero el rendimiento es notablemente superior en todos los casos, salvo para las personas con mayor educación. Esto se debe seguramente a una correlación entre tener mayor nivel educacional y la probabilidad de estar cotizando.

\section{b) Medición de conocimiento del sistema de pensiones (CSP)}

La Encuesta de Protección Social contiene asimismo más de 30 preguntas sobre el csp, incluidas en las rondas 2004, 2006 y 2009. Esto permite la construcción de un indicador de CSP, complementario del de habilidades financieras básicas (HFB).

Dado que existen ciertas diferencias en la formulación de algunas preguntas entre una ronda y otra y considerando lo encontrado por Lusardi, Mitchell y Curto (2012), en cuanto a qué diferencias en la redacción de las preguntas tienen un efecto significativo en las respuestas, se ha decidido utilizar solo las preguntas cuya formulación se mantiene inalterada para las tres rondas. Las preguntas seleccionadas son 11:

1. ¿Sabe usted qué porcentaje de su ingreso imponible le descuentan (descontaban o descontarían) mensualmente para el sistema de pensiones? - Entre un 11,1 y un $13 \%$.

2. ¿Sabe Ud. cómo se calculan las pensiones en las administradoras de fondos de pensiones (AFP)? - Considerando el saldo en cuenta individual, edad de retiro u otros elementos.

3. ¿Conoce o ha escuchado hablar del Ahorro Previsional Voluntario (APV) que opera desde el año 2002?

4. ¿Sabe Ud. cuánto hay acumulado en su cuenta individual?

5. ¿Sabe usted cuánto cobra su AFP de comisión variable por administrar sus fondos?

6. ¿Conoce o ha escuchado hablar de los multifondos?

7. ¿Sabe Ud. cuántos son los tipos de fondo que existen? -5. 
8. ¿Sabe Ud. en qué tipo de fondo están sus ahorros previsionales?

9. Según la ley, ¿a qué edad puede pensionarse el hombre? -65 años.

10. Según la ley, ¿a qué edad puede pensionarse la mujer? -60 años.

11. ¿Conoce cuáles son las distintas modalidades de pensión por vejez? —Retiro Programado, Renta Vitalicia, Renta Temporal con renta vitalicia diferida y Renta Vitalicia inmediata con retiro programado. De todas estas, las preguntas 1, 2, 7, 9, 10 y 11 son de respuesta verificable, mientras que las restantes miden declaración de conocimiento. Bravo y otros (2004, 2006 y 2008) señalan algunas discrepancias entre conocimiento autorreportado y conocimiento efectivo, sin embargo, encuentran una alta correlación entre ambos. Chan y Huff (2003) reportan que las respuestas de autodeclaración entregan información complementaria sobre la importancia y seguridad que los individuos atribuyen a la información referenciada en la pregunta. Landerretche y Martínez (2011) sugieren de todas formas considerar los resultados como cota superior del verdadero resultado en el momento de interpretar, asumiendo que el valor real es más bajo y previniendo así una posible sobreestimación de los parámetros.

Es muy importante destacar que varias de estas preguntas solo están disponibles para personas que se encuentran cotizando en el momento de ser entrevistadas. En las estimaciones de la sección siguiente se incluye también esta submuestra para poder comparar resultados entre las HFB y el csp. Tal como para el caso de las HFB, las respuestas son codificadas a fin de obtener variables binarias (correcto/incorrecto o sabe/no sabe). La fracción de respuestas correctas en porcentaje respecto de cada ronda para las diferentes categorías se encuentra en el cuadro 5 .

CUADRO 5

Conocimiento del sistema de pensiones: respuestas correctas por ronda y cohorte, 2006-2009

(En porcentajes)

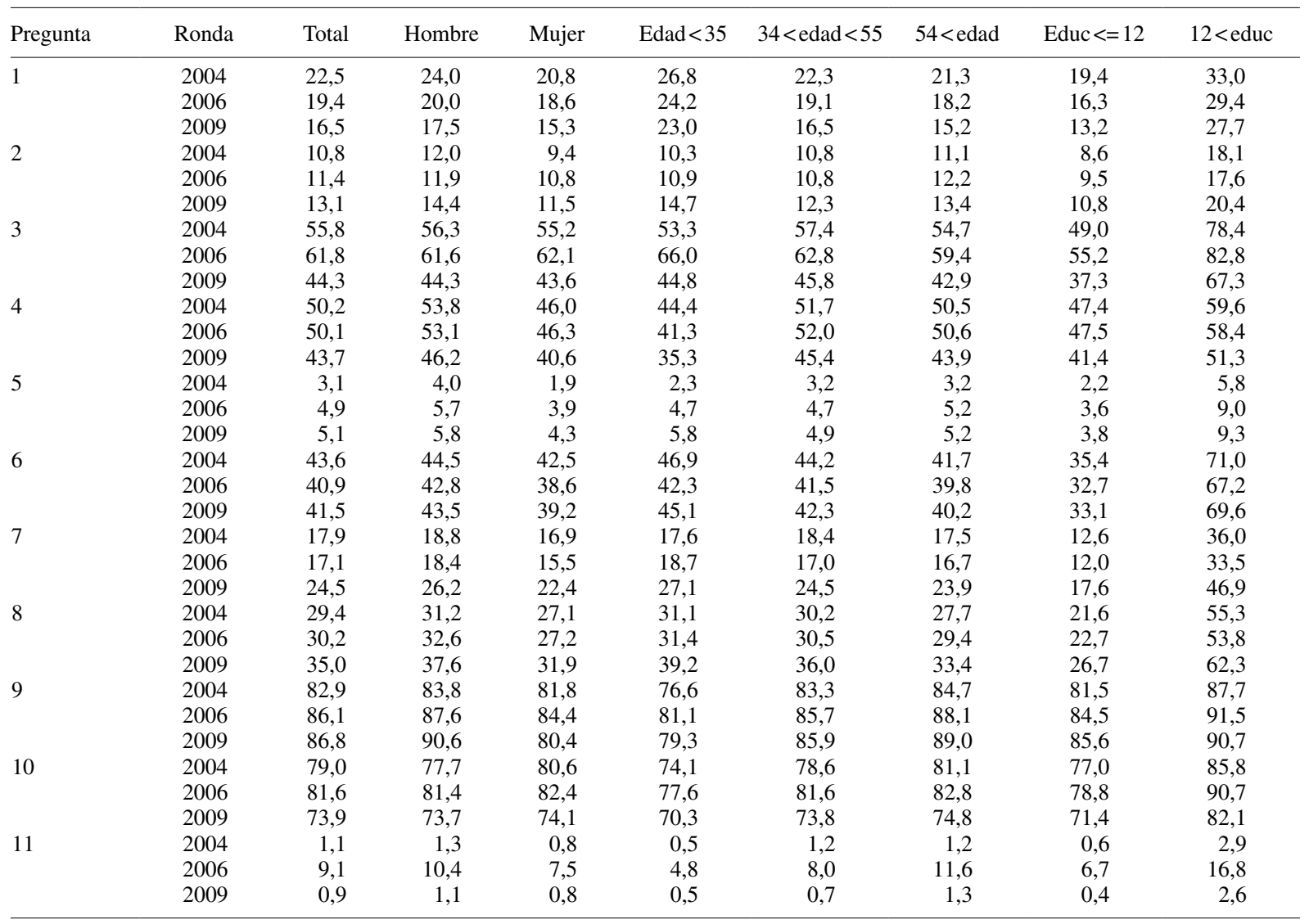

Fuente: elaboración propia sobre la base de la Encuesta de Protección Social (EPS). 
Una vez más los hombres presentan en general un mayor nivel de respuestas correctas que las mujeres en todas las preguntas de todas las rondas, con excepción de la pregunta sobre la edad de retiro de la mujer. El rendimiento por tramo etario no es tan claro ahora como en el caso anterior. Los jóvenes parecen saber mejor el porcentaje descontado para protección social y cómo están invertidos sus fondos, mientras que es la gente de mayor edad la que muestra saber mejor la edad de retiro y los diferentes sistemas de pensión. Los adultos en el tramo etario central son los que denotan saber más acerca de cómo se calculan los fondos y el monto que tienen en las cuentas. La educación aparece nuevamente como factor de diferencia relevante en los resultados, con las mayores diferencias (en torno de o levemente superiores al 30\%) en conocimiento sobre el "aporte previsional solidario" y respecto de las alternativas de inversión en los fondos de pensiones y las menores en conocimiento sobre la edad de retiro.

Con respecto al factor ronda, en general este no aparece con una tendencia clara; las preguntas 1, 2, 4 y 6 presentan los mejores resultados en la ronda de 2004; las preguntas 3, 10 y 11 aparecen con una mayor proporción de respuestas correctas en 2006, y las preguntas 5,8 y 9 evidencian un mejor rendimiento en 2009. Las diferencias, sin embargo, están por debajo de un $5 \%$ entre rondas consecutivas, salvo una caída del $20 \%$ entre 2006 y 2009 con respecto al conocimiento del APV. Cabe recordar que esto ocurre luego de balancear el panel, por lo que son los mismos individuos quienes responden en todas las rondas.

\section{c) Índices PRIDIT}

Para obtener los indicadores (HFB y CSP), las respuestas de los entrevistados en cada ronda son recodificadas mediante la aplicación de una metodología psicométrica que consiste en un análisis de componentes principales de puntajes (PRIDIT, por sus siglas en inglés) (Lieberthal, 2008). Una breve exposición de la metodología PRIDIT se encuentra en el anexo 1. Esta es una técnica no paramétrica, también utilizada por Lusardi, Mitchell y Curto (2012) en un contexto similar al presente, con el objetivo de reducir las restricciones de algunos supuestos implícitos en el promedio simple, permitiendo en particular que las respuestas inusuales tengan más peso en el indicador final (componente RIDIT), y también otorgando más peso a las preguntas cuyas respuestas más bien parecen explicar las respuestas a otras preguntas.

En el cuadro 6 se ofrece una descripción cuantitativa de los indicadores. Es importante destacar que los indicadores construidos de esta forma pueden tomar valores negativos y su valor solo es comparable en su propio contexto (los indicadores de HFB y CSP no son comparables entre sí). A objeto de ofrecer una medida de referencia, en las últimas dos columnas del cuadro 6 se entrega el promedio general de cada indicador para todas las rondas y su correspondiente desviación estándar.

Indicadores de alfabetismo financiero. Promedios por ronda y categoría, 2006-2009

\begin{tabular}{|c|c|c|c|c|c|c|c|}
\hline \multirow[t]{2}{*}{ Indicador } & \multicolumn{2}{|c|}{ HFB } & \multicolumn{2}{|c|}{ HFB (solo cotizantes) } & \multicolumn{3}{|c|}{ CSP } \\
\hline & 2006 & 2009 & 2006 & 2009 & 2004 & 2006 & 2009 \\
\hline Total & 0,0166 & $-0,0189$ & 0,1406 & 0,1138 & 0,0918 & 0,1039 & 0,0324 \\
\hline Hombres & 0,0978 & 0,0710 & 0,1857 & 0,1648 & 0,1223 & 0,1410 & 0,0792 \\
\hline Mujeres & $-0,0634$ & $-0,1050$ & 0,0840 & 0,0521 & 0,0543 & 0,0571 & $-0,0237$ \\
\hline Edad $<35$ & 0,2332 & 0,2659 & 0,2611 & 0,3015 & 0,0871 & 0,1029 & 0,0704 \\
\hline $34<$ edad $<55$ & 0,0676 & 0,0617 & 0,1417 & 0,1319 & 0,0543 & 0,0571 & $-0,0237$ \\
\hline Edad $>54$ & $-0,0716$ & $-0,1087$ & 0,1025 & 0,0634 & 0,0767 & 0,0921 & 0,0109 \\
\hline Educ $<13$ & $-0,1007$ & $-0,1296$ & 0,0105 & $-0,0038$ & $-0,0741$ & $-0,0590$ & $-0,1293$ \\
\hline Educ $>12$ & 0,4562 & 0,4426 & 0,4967 & 0,4733 & 0,5706 & 0,5496 & 0,5270 \\
\hline \multirow{2}{*}{$\begin{array}{l}\text { Media } \\
\text { Desviación estándar }\end{array}$} & \multicolumn{2}{|c|}{0,0000} & \multicolumn{2}{|c|}{0,1277} & \multicolumn{3}{|c|}{0,0777} \\
\hline & \multicolumn{2}{|c|}{0,7052} & \multicolumn{2}{|c|}{0,6936} & \multicolumn{3}{|c|}{0,7513} \\
\hline
\end{tabular}

Fuente: elaboración propia sobre la base de la Encuesta de Protección Social (EPS).

HFB: habilidades financieras básicas.

CSP: conocimiento del sistema de pensiones.

Tal como se desprende del análisis de las preguntas utilizadas, los hombres en general muestran un mayor nivel de alfabetismo financiero (AF) que las mujeres, lo que se evidencia en todos los indicadores con diferencias de entre aproximadamente 0,10 y 0,25 desviaciones estándar. El indicador de HFB pareciera ir decreciendo con la edad, mientras que el de CSP no aparece con una tendencia clara (véase el gráfico 1 (A y B)). 
GRÁFICO 1

A. Indicador de HFB promedio por tramo de edad para cada ronda (Intervalos de confianza al 10\%)

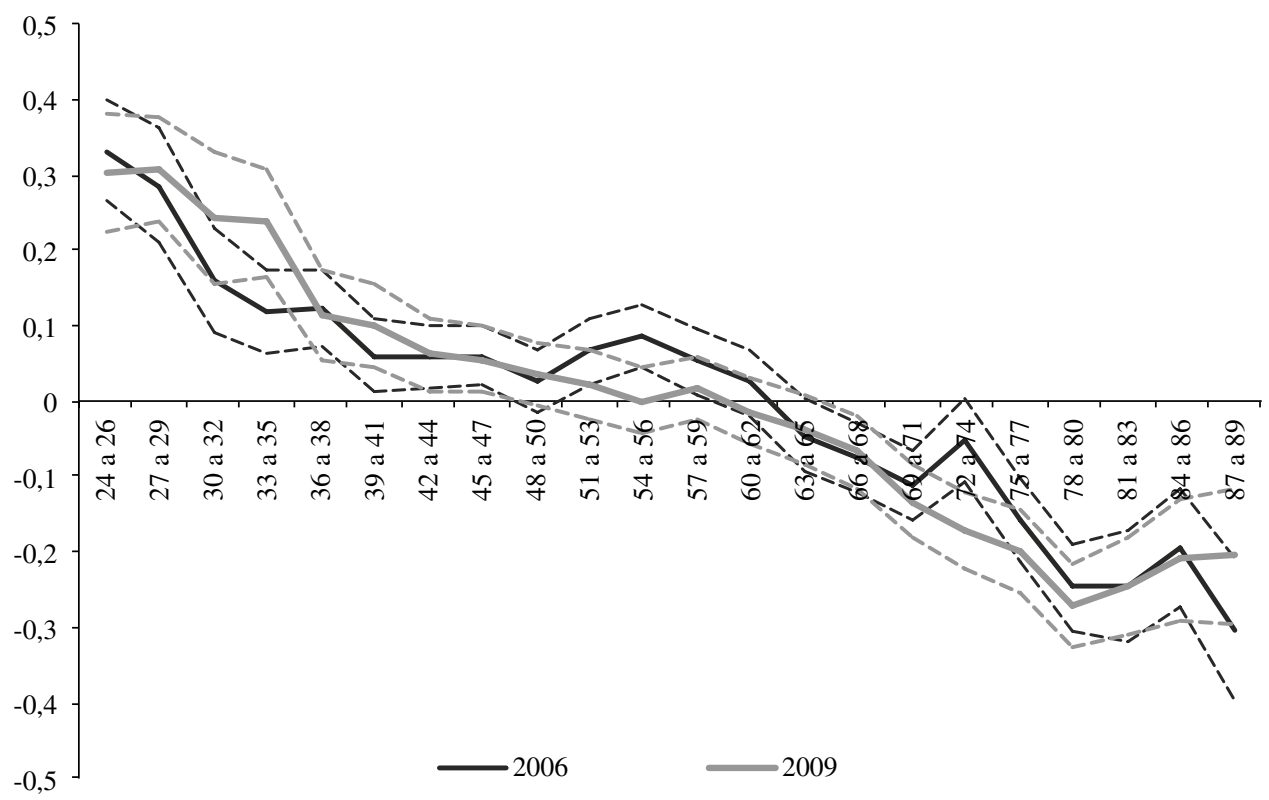

B. Indicador de CSP promedio por tramo de edad para cada ronda (Intervalos de confianza al 10\%)

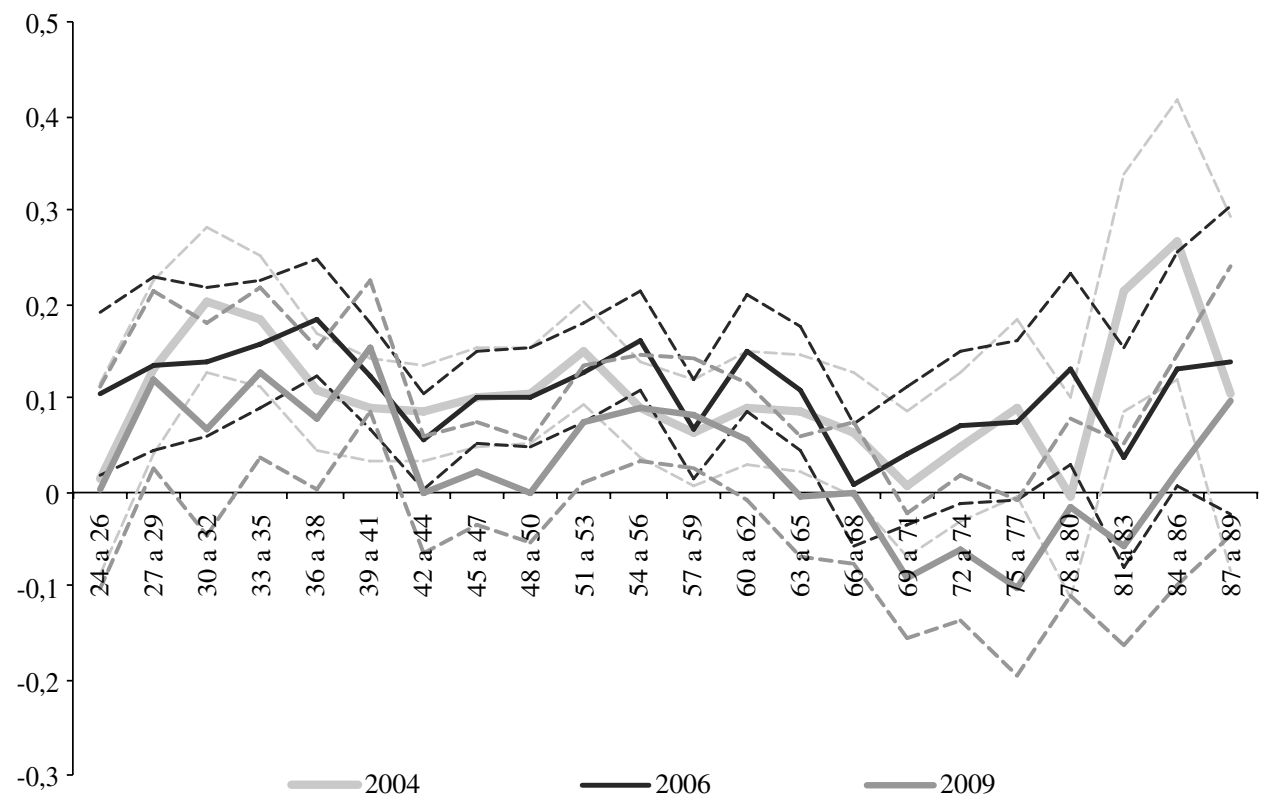

Fuente: elaboración propia sobre la base de la Encuesta de Protección Social (EPS).

HFB: habilidades financieras básicas.

CSP: conocimiento sobre el sistema de pensiones.

Nota: las líneas punteadas corresponden a los intervalos de confianza. 


\section{Análisis estadístico}

El análisis estadístico está enfocado en la comparación de resultados de un individuo en los dos indicadores de AF en rondas consecutivas. La variable dependiente es el cambio en el indicador de AF y las variables independientes son la ocurrencia o no ocurrencia de los eventos. Los 13 eventos seleccionados son incluidos simultáneamente en la misma regresión.

La regresión utilizada es nuevamente un modelo lineal de efecto fijo, de la forma:

$$
\begin{gathered}
\Delta Y_{i t}=\sum_{j=1}^{13} \beta_{j} \Delta X_{i j t}+\Delta \text { ingreso }_{i t}+\Delta \text { ingreso_hogar }_{i t} \\
+d_{\text {región it }}+d_{34}+\delta+\Delta \varepsilon_{i t}
\end{gathered}
$$

\section{III}

\section{Resultados}

Los resultados del análisis de toda la muestra (véase la primera columna del cuadro 7) resumen en buena forma lo que se observa en el análisis de las submuestras (resto de las columnas del cuadro 7): de los 13 eventos finalmente seleccionados, solo uno muestra una clara asociación con variaciones en el indicador de AF. Este evento, la capacitación laboral, denota una influencia tanto en las habilidades financieras básicas (HFB) como en el conocimiento del sistema de pensiones (CSP), y su impacto es sustantivo: de 0,271 y 0,630 en los indicadores PRIDIT de HFB y CSP, respectivamente, lo que es manifiestamente superior a la media de estos indicadores (en torno de 0,10 para ambos indicadores). Ninguno de los restantes 12 eventos es significativo.

En ninguna de las submuestras por nivel educacional se observa que algún evento tenga un impacto en el AF, con excepción del evento relativo a la capacitación laboral. donde $Y$ corresponde al indicador de conocimiento, $X$ al vector de 13 momentos receptivos potenciales, $\delta$ a la constante que captura el efecto lineal temporal,

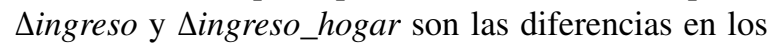
logaritmos del ingreso del entrevistado y resto del hogar respectivamente, $d_{\text {región }}$ es una variable dicotómica por región, $d_{34}$ indica si la observación es entre 2006 y $2009, i=1 . . N$ indica al individuo y $t=1,2$ corresponde al período 2006 o 2009. Se asume que para todo el resto de observables y no observables las variables se mantienen lo suficientemente fijas como para salir del modelo. El resto de los supuestos se mantienen como en las regresiones anteriores.
El análisis de las submuestras por sexo, edad y educación arroja algunos resultados adicionales, sin aportar patrones que puedan generalizarse respecto de la muestra total. El más importante de estos es el impacto de cambios en la salud entre las mujeres y en los individuos menores de 54 años. Sin embargo, en estas submuestras los eventos de salud evidencian una influencia positiva en las HFB y negativa en el CSP. Es posible que ello se deba a divergencias en las dinámicas de apreciación y depreciación del acervo de AF del individuo, o a procesos de inserción y desinserción en la fuerza laboral, entre otras posibles explicaciones.

En el presente estudio, todas las regresiones han sido repetidas mediante la utilización de indicadores calculados como promedios simples, en lugar de un análisis de componentes principales de puntajes PRIDIT, obteniéndose resultados similares. 
Resultados de las regresiones

\begin{tabular}{|c|c|c|c|c|c|c|c|c|}
\hline Submuestra & $\begin{array}{c}\text { Total de la } \\
\text { muestra }\end{array}$ & Hombres & Mujeres & $\begin{array}{c}\text { Menores de } \\
35 \text { años }\end{array}$ & $\begin{array}{c}\text { Entre } 35 \text { y } \\
54 \text { años }\end{array}$ & $\begin{array}{c}\text { Mayores de } \\
54 \text { años }\end{array}$ & $\begin{array}{c}\text { Hasta } 12 \\
\text { años de } \\
\text { escolaridad }\end{array}$ & $\begin{array}{c}\text { Más de } 12 \\
\text { años de } \\
\text { escolaridad }\end{array}$ \\
\hline
\end{tabular}

\begin{tabular}{|c|c|c|c|c|c|c|c|c|}
\hline \multirow{2}{*}{$\begin{array}{l}\text { Evento } \\
\text { Tener hijos }\end{array}$} & \multicolumn{8}{|c|}{ Indicador de HFB con toda la muestra } \\
\hline & $0,154 * * *$ & 0,090 & $0,199 * * *$ & 0,066 & 0,057 & $0,267 * * *$ & $0,161 * *$ & 0,018 \\
\hline Casarse & 0,053 & 0,086 & 0,013 & $-0,019$ & 0,066 & 0,055 & 0,040 & 0,054 \\
\hline Enviudar & $-0,187$ & $-0,461$ & $-0,069$ & $0,820 * * *$ & $-0,427$ & $-0,149$ & $-0,120$ & $-0,130$ \\
\hline Obtener un título profesional & 0,180 & 0,045 & $0,274^{*}$ & $0,350 * *$ & $-0,220$ & $-0,003$ & - & $-0,231 *$ \\
\hline Obtener un diploma & $0,129 *$ & 0,055 & 0,162 & $-0,277$ & 0,116 & $0,368 * *$ & $0,195 * *$ & $-0,255^{* *}$ \\
\hline Capacitación laboral & $0,363 * * *$ & $0,334 * * *$ & $0,360 * * *$ & $0,290 * *$ & $0,288 * * *$ & $0,470 * * *$ & $0,297 * * *$ & 0,081 \\
\hline Aprender un oficio & 0,034 & $0,211 *$ & $-0,131$ & 0,075 & $-0,032$ & 0,130 & 0,024 & $-0,093$ \\
\hline $\begin{array}{l}\text { Obtener el primer trabajo } \\
\text { permanente }\end{array}$ & $-0,060$ & $-0,030$ & $-0,052$ & $-0,096$ & $-0,096$ & $-0,083$ & $-0,033$ & 0,030 \\
\hline Jubilar & $-0,287 * * *$ & $-0,299$ & $-0,253 * * *$ & - & $-0,704 * * *$ & $-0,200 * * *$ & $-0,222 * * *$ & $-0,039$ \\
\hline Quedar discapacitado & $-0,148 * *$ & $0,008 * * *$ & $-0,255^{* * *}$ & 0,059 & $-0,032$ & $-0,174 * *$ & $-0,145 * *$ & 0,128 \\
\hline Dejar de ser discapacitado & $-0,056$ & $-0,030$ & $-0,079$ & $0,902 * * *$ & 0,037 & $-0,068$ & 0,002 & $-0,109$ \\
\hline Mejora en salud & 0,105 & $-0,331 * *$ & $0,426 * * *$ & $0,560 * * *$ & $0,303 * *$ & $-0,125$ & 0,181 & $0,450 *$ \\
\hline Empeoramiento de salud & 0,029 & $-0,032$ & 0,042 & 0,024 & $-0,016$ & 0,044 & 0,065 & $-0,110$ \\
\hline Evento & \multicolumn{8}{|c|}{ Indicador de HFB solo con los cotizantes } \\
\hline Tener hijos & $0,104^{*}$ & 0,052 & $0,139 *$ & 0,075 & 0,013 & $0,198 * *$ & $0,114^{*}$ & 0,010 \\
\hline Casarse & 0,027 & 0,012 & 0,045 & $-0,140$ & 0,099 & 0,009 & 0,005 & 0,027 \\
\hline Enviudar & $-0,389$ & $-0,116$ & $-0,493$ & $0,758 * * *$ & $-0,918 * * *$ & $-0,509 * *$ & $-0,392$ & $-0,256$ \\
\hline Obtener un título profesional & $0,196^{*}$ & 0,000 & $0,325 * * *$ & $0,358 * *$ & $-0,334 * *$ & 0,177 & - & $-0,166$ \\
\hline Obtener un diploma & 0,059 & 0,019 & 0,083 & $-0,411 * * *$ & 0,110 & 0,241 & 0,129 & $-0,273 * *$ \\
\hline Capacitación laboral & $0,271 * * *$ & $0,263 * * *$ & $0,274 * * *$ & $0,212 *$ & $0,228 * * *$ & $0,338 * * *$ & $0,212 * * *$ & 0,055 \\
\hline Aprender un oficio & $-0,034$ & $0,252 * *$ & $-0,248 * *$ & $-0,001$ & $-0,141$ & 0,138 & $-0,020$ & $-0,121$ \\
\hline $\begin{array}{l}\text { Obtener el primer trabajo } \\
\text { permanente }\end{array}$ & $-0,066$ & $-0,038$ & $-0,067$ & $-0,193$ & $-0,063$ & $-0,032$ & $-0,073$ & 0,101 \\
\hline Jubilar & $-0,280 * *$ & $-0,431 * * *$ & 0,163 & - & $-0,942 * * *$ & $-0,189 *$ & $-0,195^{*}$ & $-0,250$ \\
\hline Quedar discapacitado & 0,039 & 0,091 & 0,003 & $-0,031$ & 0,120 & $-0,011$ & 0,028 & 0,115 \\
\hline Dejar de ser discapacitado & $0,196 * *$ & 0,188 & 0,225 & $0,776^{* * *}$ & $0,205^{*}$ & 0,156 & $0,217 * *$ & 0,241 \\
\hline Mejora en salud & 0,057 & $-0,361 * *$ & $0,325^{*}$ & $0,517 * * *$ & $0,348 * *$ & $-0,305$ & 0,142 & $0,432 *$ \\
\hline Empeoramiento de salud & 0,045 & $-0,089$ & 0,163 & 0,091 & 0,008 & 0,041 & 0,051 & 0,020 \\
\hline Evento & \multicolumn{8}{|c|}{ Indicador de CSP } \\
\hline Tener hijos & 0,087 & 0,039 & 0,125 & $-0,026$ & 0,058 & $0,249 * *$ & 0,105 & $-0,020$ \\
\hline Casarse & 0,124 & 0,116 & 0,105 & 0,207 & $-0,048$ & 0,207 & 0,122 & 0,060 \\
\hline Enviudar & $-0,489$ & $-0,227$ & $-0,613 * *$ & $0,988 * * *$ & $-0,735^{* * *}$ & $-0,635^{*}$ & $-0,453$ & $-0,594 * * *$ \\
\hline Obtener un título profesional & $-0,004$ & 0,105 & $-0,062$ & $-0,212$ & $-0,166$ & 0,256 & - & $-0,444 * * *$ \\
\hline Obtener un diploma & $0,392 * * *$ & $0,546^{* * *}$ & $0,259 * *$ & $0,315^{*}$ & $0,336 * * *$ & $0,585 * * *$ & $0,341 * * *$ & $0,184 * *$ \\
\hline Capacitación laboral & $0,630 * * *$ & $0,646^{* * *} *$ & $0,623 * * *$ & $0,709 * * *$ & $0,624 * * *$ & $0,607 * * *$ & $0,609 * * *$ & $0,301 * * *$ \\
\hline Aprender un oficio & $0,241 * * *$ & $0,289 * *$ & 0,188 & 0,001 & 0,161 & $0,468 * * *$ & $0,249 * * *$ & 0,138 \\
\hline $\begin{array}{l}\text { Obtener el primer trabajo } \\
\text { permanente }\end{array}$ & $-0,214 * *$ & $-0,015$ & $-0,325 * * *$ & $-0,141$ & $-0,391 * * *$ & $-0,016$ & $-0,226 * * *$ & $-0,023$ \\
\hline Jubilar & $-0,209$ & $-0,210$ & $-0,155$ & - & $-0,743 * * *$ & $-0,155$ & $-0,071$ & $-0,436$ \\
\hline Quedar discapacitado & $-0,211 * *$ & $-0,147$ & $-0,308 * *$ & $-0,108$ & 0,013 & $-0,431 * * *$ & $-0,224 * * *$ & $-0,108$ \\
\hline Dejar de ser discapacitado & $-0,148$ & $-0,130$ & $-0,183$ & 0,000 & $-0,239 *$ & $-0,089$ & $-0,132$ & 0,042 \\
\hline Mejora en salud & $-0,343 * * *$ & $-0,407 * *$ & $-0,285^{*}$ & $-0,861 * * *$ & $-0,349 * *$ & $-0,282$ & $-0,206^{* *}$ & $-0,348$ \\
\hline Empeoramiento de salud & 0,099 & $-0,067$ & 0,232 & $0,537 * * *$ & 0,168 & $-0,114$ & 0,118 & 0,098 \\
\hline
\end{tabular}

Fuente: elaboración propia sobre la base de la Encuesta de Protección Social (EPS).

HFB: habilidades financieras básicas.

CSP: conocimiento sobre el sistema de pensiones.

$*$ significativo al $10 \%$; ** significativo al $5 \%$; *** significativo al $1 \%$. 


\section{IV}

\section{Discusión}

Dada la importancia que se suele atribuir a la idea del AF en lo que se refiere a su repercusión en el bienestar, y motivados por diversos estudios que muestran en general un nivel de alfabetización financiera (AF) en la población, los gobiernos han impulsado diversos programas de AF. No obstante, en la literatura no existe consenso respecto de la efectividad de los programas y la robustez del marco conceptual del AF.

En la conceptualización más común (aquí llamada "modelo económico" del AF), los individuos deciden cuánto AF adquirir en función de los beneficios esperados de este en el proceso de toma de decisiones. Sin embargo, en este estudio el modelo económico del AF resultó poco ajustado a los datos. En efecto, no se encontró evidencia contundente de un aumento en el nivel de AF en personas que experimentaron eventos que implican cambios en su situación financiera. Esta conclusión se obtiene al analizar conjunta y separadamente dos indicadores alternativos de AF, y sobre la base de un panel de más de 12.000 mil individuos encuestados un máximo de cuatro veces en un período de siete años, y segregando la muestra en varias submuestras.

ANEXO 1

\section{PRIDIT}

PRIDIT es una técnica de agregación no paramétrica, que consiste en la utilización de dos procedimientos para jerarquizar muestras según observables categóricos: un análisis de componente principal de puntajes RIDIT (Lieberthal, 2008).

RIDIT es una metodología desarrollada para analizar variables categóricas - en este caso binariasutilizadas como proxy para no observables (Lieberthal, 2008). La idea subyacente en la aplicación de RIDIT en este estudio es que una respuesta incorrecta puede ser más informativa acerca del AF de una persona que una respuesta correcta, y viceversa. Ello porque en algunas preguntas la mayoría de los individuos responden correctamente y entonces la respuesta incorrecta es la que identifica a un grupo especial de individuos, mientras que en preguntas donde lo común es errar, la respuesta correcta es la más informativa.
En suma, no es claro que el modelo económico sea un buen modelo para el AF. Más allá de las críticas que pueden hacerse a este trabajo en relación con la calidad de los datos y de los indicadores de AF, y también sobre la validez de la estrategia empírica, el presente estudio se suma a un grupo de trabajos que, por una u otra razón, han cuestionado la solidez conceptual del AF.

Es posible que el AF no pueda reducirse a un concepto simple. Incluso en un contexto más general, los bienes de información (information goods) presentan considerables complejidades (Bates, 1990; Rafaeli y Raban, 2003). También puede suceder que el AF se entienda como una característica del individuo que no cambia en el corto plazo, tal como la inteligencia. Existe un modelo de inteligencia fluida versus inteligencia cristalizada que contribuiría a comprender una eventual asociación entre el AF y la edad (Agarwal y otros, 2009). O quizás el AF corresponda a una actitud, antes que a un conocimiento. O también, y sin perjuicio de lo anterior, que los individuos actualicen su AF, depreciándolo y apreciando en parte, de tal forma que la variación neta del AF sea normalmente muy pequeña.

Asignar unos y ceros a todas las respuestas correctas e incorrectas por igual para construir el indicador presupone, primero, que el alfabetismo financiero es métricamente medible - supuesto que no discutiremos-, y segundo, que la métrica puede ser escalada con intervalos equidistantes entre las respuestas de cada pregunta en la encuesta (Brockett y otros, 2002). RIDIT da cuenta de este problema utilizando información muestreal en cada pregunta para asignar distintos valores o pesos a las respuestas (Lieberthal, 2008).

En la práctica, siguiendo a Brockett y otros (2002), para construir los puntajes RIDIT en este trabajo se utilizó el siguiente algoritmo: sea $\hat{p}_{t i}$ la probabilidad muestral de obtener una respuesta $i$ para la pregunta $t$, donde $i=0,1$ es la cantidad de categorías de la respuesta $t$. Se definen entonces los puntajes RIDIT como:

$$
R_{t i}=\sum_{j<i} \hat{p}_{t j}-\sum_{j>i} \hat{p}_{t j}
$$


Así, en lugar de asignar ceros y unos, se asignan $R_{t 0}$ y $R_{t 1}$ a las respuestas de cada pregunta $t$. Este puntaje es monotónicamente creciente en las categorías, manteniendo la clasificación original y cumpliéndose además que $E\left(R_{t}\right)=0$. En palabras de Brockett y otros (2002), con este método no es necesario asignar valores enteros de modo ad hoc al tiempo que mejoran las características estadísticas de los datos evaluados resultantes para el subsecuente análisis estadístico estándar, sea este cual sea (Brockett y otros, 2002).

PRIDIT: una vez obtenidos los puntajes RIDIT para cada pregunta, el análisis de componente principal se hace cargo de repartir pesos entre las preguntas, ponderando según la importancia de la pregunta en la varianza de las notas finales. Utiliza un algoritmo convergente que computa las ponderaciones, de manera tal que a las preguntas menos correlacionadas con una combinación lineal de las otras preguntas se les asigna un peso mayor, pues estas son más informativas. En otras palabras, las respuestas más “extrañas" en comparación con las demás respuestas adquieren mayor atención a la hora de computar el puntaje final (Lusardi, Mitchell y Curto, 2012).

\section{Bibliografía}

Agarwal, S. y otros (2009), "The age of reason: Financial decisions over the life-cycle with implications for regulation", Brookings Papers of Economic Activities, vol. 40, $\mathrm{N}^{\circ}$ 2, Washington, D.C., The Brookings Institution.

Bates, B.J. (1990), "Information as an economic good: A re-evaluation of theoretical approaches", Mediation, Information, and Communication. Information and Behavior, B.D. Ruben y L.A. Lievrouw (eds.), New Brunswick, Transaction Publishers.

Bravo, D. y otros (2008), Determinantes del Ahorro Previsional Voluntario en Chile, Santiago de Chile, Centro de Microdatos, Departamento de Economía, Universidad de Chile.

(2006), "Encuesta de Protección Social 2004: Presentación general y principales resultados" [en línea] http://www. previsionsocial.gob.cl/subprev/?wpfb_dl=17.

(2004), Análisis y principales resultados. Primera Encuesta de Protección Social. Historia laboral y seguridad social, Santiago de Chile, Universidad de Chile.

Brockett, P.L. y otros (2002), "Fraud classification using principal component analysis of RIDITs", The Journal of Risk and Insurance, vol. 69, $\mathrm{N}^{\circ} 3$, Wiley.

Campbell, J.Y. y otros (2011), "Consumer financial protection", Journal of Economic Perspectives, vol. 25, $\mathrm{N}^{\circ} 1$, Nashville, Tennessee, American Economic Association.

Chan, S. y A. Huff (2003), "What you don't know can't help you: Pension knowledge and retirement decision making", NBER Working Paper, $\mathrm{N}^{\circ} 10185$, Cambridge, Massachusetts, National Bureau of Economic Research.

Christelis, D., T. Jappelli y M. Padula (2005), "Health risk, financial information and social interactions: The portfolio choice by European elderly households", Documento de Trabajo, Universidad de Salerno.

Delavande, A., S. Rohwedder y R. Willis (2008), "Preparation for retirement, financial literacy and cognitive resources", Working Paper, $\mathrm{N}^{\circ} 190$, Michigan, Universidad de Michigan.

Demark-Wahnefried, W. y otros (2005), "Riding the crest of the teachable moment: Promoting long-term health after the diagnosis of cancer", Journal of Clinical Oncology, vol. 23, $\mathrm{N}^{\circ} 24$, Nueva York, American Society of Clinical Oncology.

Fajnzylber, E., G. Plaza y G. Reyes (2009), "Better-informed workers and retirement savings decisions: Impact evaluation of a personalized pension projection in Chile", Documento de Trabajo, $\mathrm{N}^{\mathrm{o}}$ 31, Santiago de Chile, Superintendencia de Pensiones.

GAO (Oficina de Rendición de Cuentas del Gobierno) (2004), "Highlights of a GaO Forum: The Federal Government's Role in Improving Financial Literacy" [en línea] http://www.gao. gov/products/GAO-05-93SP
Gerardi, K., L. Goette y S. Meier (2010), "Financial literacy and subprime mortgage delinquency: Evidence from a survey matched to administrative data", Working Paper Series, $\mathrm{N}^{\circ}$ 2010-10, Atlanta, Banco de la Reserva Federal de Atlanta.

Hansen, E.J. (1998), "Creating teachable moments... and making them last", Innovative Higher Education, vol. $23, \mathrm{~N}^{\circ} 1$, Springer.

Hastings, J. y L. Tejeda-Ashton (2008), "Financial literacy, information, and demand elasticity: Survey and experimental evidence from Mexico", NBER Working Paper, $\mathrm{N}^{\circ} 14538$, Cambridge, Massachusetts, National Bureau of Economic Research.

Hathaway, I. y S. Khatiwada (2008), "Do financial education programs work?", Working Paper, № 08-03, Cleveland, Banco de la Reserva Federal de Cleveland.

Hilgert, M.A., J.M. Hogarth y S.G. Beverly (2003), "Household financial management: The connection between knowledge and behavior", Federal Reserve Bulletin, vol. 89, $\mathrm{N}^{\circ} 7$.

Hogarth, J. y M. Hilgert (2002), "Financial knowledge, experience and learning preferences: Preliminary results from a new survey on financial literacy", Consumer Interests Annual, vol. 48, $\mathrm{N}^{\circ} 1$.

Huston, S.J. (2010), "Measuring financial literacy", Journal of Consumer Affairs, vol. 44, $\mathrm{N}^{\circ} 2$, Wiley.

Landerretche, O. y C. Martínez (2011), "Voluntary savings, financial behavior and pension finance literacy: Evidence from Chile", Documento de Trabajo, $\mathrm{N}^{\circ} 328$, Santiago de Chile, Departamento de Economía, Universidad de Chile.

Lieberthal, R.D. (2008), "Hospital quality: A PRIDIT approach", Health Services Research, vol. 43, $\mathrm{N}^{\circ} 3$, Chicago, Health Research \& Educational Trust, junio.

Liker, J.K., S. Augustyniak y G.J. Duncan (1985), "Panel data and models of change: A comparison of first difference and conventional two wave models", Social Science Research, vol. 14, $\mathrm{N}^{\circ} 1$, Amsterdam, Elsevier.

Lusardi, A. (2008), "Financial literacy: An essential tool for informed consumer choice?", CFS Working Paper Series, № 19, Center for Financial Studies.

Lusardi, A. y O. Mitchell (2007a), "Baby boomer retirement security: The role of planning, financial literacy, and housing wealth", Journal of Monetary Economics, vol. 54, $\mathrm{N}^{\circ} 1$, Amsterdam, Elsevier.

(2007b), "Financial literacy and retirement preparedness: Evidence and implications for financial education", Business Economics, vol. $42, \mathrm{~N}^{\circ} 1$.

(2006), "Financial literacy and planning: Implications for retirement and wellbeing", DNB Working Paper, $\mathrm{N}^{\circ} 078$, Netherlands Bank. 
Lusardi A., O. Mitchell y V. Curto (2012), "Financial sophistication in the older population", NBER Working Paper, $\mathrm{N}^{\circ} 17863$, Cambridge, Massachusetts, National Bureau of Economic Research.

(2010), "Financial literacy among the young", Journal of Consumer Affairs, Special Issue on Financial Literacy, vol. 44, $\mathrm{N}^{\circ} 2$, Wiley.

Lyons, A.C. y otros (2006), "Are we making the grade? A national overview of financial education and program evaluation", Journal of Consumer Affairs, vol. 40, $\mathrm{N}^{\circ} 2$, Wiley.

Mason, C. y R. Wilson (2000), "Conceptualizing financial literacy", Research Series Paper, $\mathrm{N}^{\circ}$ 7, Londres, Business School, Universidad de Laughborough.

Mandell, L. y L.S. Klein (2009), "The impact of financial literacy education on subsequent financial behavior", Journal of Financial Counseling and Planning, vol. 20, $\mathrm{N}^{\circ} 1$.

(2007), "Motivation and financial literacy", Financial Services Review, vol. 16, $\mathrm{N}^{\circ} 2$.

McBride, C.M., K.M. Emmons e I.M. Lipkus (2003), "Understanding the potential of teachable moments: The case of smoking cessation", Health Education Research, vol. 18, $\mathrm{N}^{\circ}$ 2, Oxford, Oxford University Press.

McBride, C.M. y J.S. Ostroff (2003), "Teachable moments for promoting smoking cessation: the context of cancer care and survivorship", Cancer Control, vol. 10, $\mathrm{N}^{\circ} 4$.

Miles, D. (2004), The UK Mortgage Market: Taking a Longer-Term View", Londres, The Stationery Office.
Rafaeli, S. y D.R. Raban (2003), "Experimental investigation of the subjective value of information in trading", Journal of the Association for Information Systems, vol. 4, $\mathrm{N}^{\circ} 1$.

Remund, D.L. (2010), "Financial literacy explicated: the case for a clearer definition in an increasingly complex economy", Journal of Consumer Affairs, vol. 44, $\mathrm{N}^{\circ}$ 2, Wiley.

Servon, L.J. y R. Kaestnert (2008), "Consumer financial literacy and the impact of online banking on the financial behavior of lower-income bank customers", Journal of Consumer Affairs, vol. $42, \mathrm{~N}^{\circ} 2$, Wiley.

Stone, L.O. y B. Neumann (2012), "Gender differences in preparedness to meet retirement's challenges", Key Demographics in Retirement Risk Management, L. Stone (ed.), Springer.

Syvertzen, A.K., M.D. Stout y C.A. Flanagan (2009), "Using elections as teachable moments: A randomized evaluation of the student voices civic education programs", American Journal of Education, vol. 116, $\mathrm{N}^{\circ}$ 1, Chicago, University of Chicago Press.

Van Rooij, M., A. Lusardi y R. Alessie (2011), "Financial literacy and stock marker participation", Journal of Financial Economics, vol. 101, $\mathrm{N}^{\circ} 2$, Amsterdam, Elsevier.

Willis, L.E. (2009), "Evidence and ideology in assessing the effectiveness of financial literacy education", San Diego Law Review, vol. 46, San Diego, Universidad de San Diego.

(2008), "Against financial-literacy education", Iowa Law Review, vol. 94, Iowa. 